\title{
Weed Phytosociological and Floristic Survey in Agricultural AREAS OF SOUTHWESTERN GOIÁs REgION ${ }^{1}$
}

\author{
Levantamento Fitossociológico e Florístico de Plantas Daninhas em Áreas Agrícolas da Região \\ Sudoeste de Goiás
}

SANTOS, W.F. ${ }^{2}$, PROCÓPIO, S.O. ${ }^{3}$, SILVA, A.G. ${ }^{2}$, FERNANDES, M.F. ${ }^{3}$, and BARROSO, A.L.L. ${ }^{2}$

\begin{abstract}
The use of the Roundup Ready ${ }^{\circledR}$ technology and the cultivation of a second crop influence the floristic composition of weed communities in Brazilian Central-West region cropping systems. This study has aimed to diagnose the dominant weed species in southwestern Goiás in areas of genetically-modified and conventional soybeans, using phytosociological and floristic surveys. Weed sampling was obtained by collecting all the plants present within a $0.5 \mathrm{~m}$ hollow frame, randomly thrown 20 times in each of thirty-five agricultural areas in the 2012/2013 harvest. Field survey was carried out in three periods: before desiccation for soybean sowing, before postemergence herbicide in soybean first application and before postemergence herbicide application in late harvest. A total of $525 \mathrm{~m}^{2}$ was inventoried and 3,219 weeds were collected, which included 79 species, 58 genera and 28 families. Families Poaceae, Asteraceae, Euphorbiaceae, Fabaceae, Amaranthaceae, were the most representative in the survey. Species Cenchrus echinatus, Glycine max, Chamaesyce hirta, Commelina benghalensis, and Alternanthera tenella stood out in importance. The RR+millet soybean treatment had the highest number of species (44), while the conventional soybean + sorghum treatment had the lowest number of species (18). The highest number of species was recorded in first sampling period. Treatments conventional soybean + maize and conventional soybean + millet showed higher similarity $(70 \%)$, while treatments RR soybean + millet and conventional soybean + sorghum showed the least (51\%). Species of difficult control were recorded in all cultivation systems analyzed.
\end{abstract}

Keywords: phytosociology, floristic, soybean, late harvest, importance value.

RESUMO - A utilização da tecnologia Roundup Ready ${ }^{\mathbb{B}}$ e o cultivo de segunda safra influenciam a composição florística das comunidades infestantes dos sistemas de cultivo do Centro-Oeste. Este estudo objetivou diagnosticar as espécies de plantas daninhas predominantes na região sudoeste de Goiás, em áreas de soja geneticamente modificada e soja convencional, por meio de levantamento fitossociológico e florístico. Para amostragem das plantas daninhas foi utilizado um quadrado vazado de 0,5 m, lançado aleatoriamente 20 vezes em cada uma das 35 áreas agrícolas na safra 2012/ 2013. O levantamento de campo foi realizado em três épocas: antes da dessecação para o plantio da soja, previamente à primeira aplicação de herbicida em pós-emergência da soja e anteriormente à aplicação de pós-emergência da safrinha. Foram inventariados $525 \mathrm{~m}^{2}$ no total, sendo coletadas 3.219 plantas daninhas, distribuidas em 79 espécies, 58 gêneros e 28 famílias. As famílias Poaceae, Asteraceae, Euphorbiaceae, Fabaceae e Amaranthaceae, foram as mais representativas. As espécies Cenchrus echinatus, Glycine max, Chamaesyce hirta, Commelina benghalensis $e$ Alternanthera tenella destacaram-se em valor de importância. O tratamento soja $R R+$ milheto apresentou o maior número de espécies (44), e o tratamento soja convencional+sorgo, o menor (18). Registrou-se o maior número de espécies na primeira época de avaliação. Os tratamentos soja convencional+milho e soja convencional + milheto mostraram maior similaridade (70\%), enquanto os tratamentos soja $R R+$ milheto e soja convencional + sorgo apresentaram a menor (51\%). Espécies de dificil controle foram registradas em todos os sistemas cultivo de analisados.

Palavras-chave: fitossociologia, florística, soja, safrinha, valor de importância.

Recebido para publicação em 13.8.2015 e aprovado em 26.8.2015.

2 Universidade de Rio Verde/Programa de Pós-graduação em Produção Vegetal/Faculdade de Agronomia, Rio Verde-GO, Brasil, $<$ santoswf1@gmail.com>; ${ }^{3}$ Embrapa Tabuleiros Costeiros, Aracaju-SE, Brasil. 


\section{INTRODUCTION}

The cultivation of glyphosate-tolerant soybeans significantly alters the chemical control mechanisms in Brazil. In recent decades, this has been the main weed control tool in Brazilian agroecosystems (Balbinot Jr. \& Veiga, 2014).

The expansion of the areas that use Roundup Ready ${ }^{\mathbb{B}}(\mathrm{RR})$ soybeans associated with second crop cultivation are notably the main changes diagnosed in agricultural systems focused on grain production. These changes have a direct influence on the weeds population dynamics in different crop rotation/succession systems.

After the release of the RR soybean in the 2003/04 harvest, there was a rapid growth of the cultivated area with such materials, increasing the use of glyphosate across the country (Fialho et al., 2011). Several reasons are given to justify the rapid adoption of this technology: 1) broad spectrum of action; 2) action in both early and late postemergence; 3) simplification of the process of choosing herbicides; 4) decrease in the occurrence of visual symptoms in soybean plants; 5) no carryover problems; and 6) control of perennial or annual species (Barros et al., 2014).

Prior identification of weeds is the first step in planning the best control methods in cropping systems. The highest population densities and the most harmful species in agricultural areas must be observed, from conducting floristic and phytosociological studies (Pitelli, 2000; Cruz et al., 2009).

The presence of weeds in agricultural areas can cause losses in grain yield, mainly due to competition for water, light and nutrients. Most often, these growth factors are insufficient even for the development of the main culture (Radosevich et al., 1997).

Southwestern Goiás has become one of the main responsible areas for the domestic growth of grain production. Soybeans cultivation in the harvest and maize, sorghum and millet in the late harvest have included this region in the economic dynamics of the country. Conducting research that support these production systems is critical to increased agricultural expansion, making the agroecosystems more efficient and balanced.

The expansion of direct seeding and increased use of RR soybeans have modified the management mechanisms and chemical control in southwestern Goiás. These changes influence the floristic composition and population dynamics of weeds. However, few studies have been conducted about the weed communities in this region.

Considering the changes in southwestern Goiás cropping systems, this study has aimed to diagnose and compare the predominant weed species in agricultural areas which grow glyphosate-tolerant soybeans and conventional soybeans in the first season with different crops in late harvest.

\section{MATERIAL AND METHODS}

The areas of this study are located in southwestern Goiás farms. Field surveys have covered Brazilian municipalities Rio Verde, Santa Helena de Goiás, Santo Antônio da Barra, and Montividiu, in the 2012/2013 harvest between the months of June 2012 and July 2013.

Regarding the Köppen-Geiger classification, the studied municipalities have an Aw climate, with average temperatures of 23.0 to $24.3{ }^{\circ} \mathrm{C}$ and average annual rainfall of 1510-1663 $\mathrm{mm}$, with the highest concentration in the summer. Winter are dry, with mild temperatures and no rain between the months of May and September. The soils of the region are types dystrophic red latosol and dystrophic red latosol (Santos et al., 2011).

Field surveys took place in seven production systems (treatments), with five replications in different properties that had at least three consecutive years of implementation, totaling 35 agricultural areas (Table 1). In these properties, soybeans in the harvest and maize, sorghum and millet or fallow after the main harvest (succession or late harvest) predominate.

Field survey was conducted in three evaluation periods: before desiccation for soybean crop implementation; prior to the first application of postemergence herbicides in the soybean crop, at 20 days after sowing; and 
Table 1 - Location of properties conducting a weeds survey in southwestern Goiás

\begin{tabular}{|c|c|c|c|c|c|c|}
\hline \multicolumn{3}{|c|}{ Treatment } & \multirow[b]{2}{*}{$\begin{array}{c}\text { Areas } \\
\text { (Replications) }\end{array}$} & \multirow{2}{*}{$\begin{array}{c}\text { Location } \\
\text { Coordinates } \\
\text { (Universal Transversa de } \\
\text { Mercator (UTM)) }\end{array}$} & \multirow[b]{2}{*}{$\begin{array}{l}\text { Height } \\
\text { (m) }\end{array}$} & \multirow[b]{2}{*}{ Municipalities/Goiás } \\
\hline № & $\begin{array}{l}\text { Soybeans/ } \\
\text { summer }\end{array}$ & Late harvest & & & & \\
\hline 1 & RR Soybean & RR Maize & 1 & $22 \mathrm{k} 482613.93 / 8100304.26$ & 862 & Montividiu \\
\hline 1 & RR Soybean & RR Maize & 2 & 22 k 540809.09/8008785.91 & 639 & Rio Verde \\
\hline 1 & RR Soybean & RR Maize & 3 & $22 \mathrm{k} 524462.03 / 8019154.20$ & 635 & Rio Verde \\
\hline 1 & RR Soybean & RR Maize & 4 & $22 \mathrm{k} 560434.78 / 8044516.10$ & 557 & Santa Helena \\
\hline 1 & RR Soybean & RR Maize & 5 & $22 \mathrm{k} 528012.52 / 8021719.00$ & 627 & Rio Verde \\
\hline 2 & RR Soybean & Millet & 1 & $22 \mathrm{k} 503225.37 / 8077848.74$ & 828 & Rio Verde \\
\hline 2 & RR Soybean & Millet & 2 & $22 \mathrm{k} 505769.78 / 8079871.48$ & 799 & Montividiu \\
\hline 2 & RR Soybean & Millet & 3 & $22 \mathrm{k} 506350.89 / 8079876.84$ & 768 & Montividiu \\
\hline 2 & RR Soybean & Millet & 4 & $22 \mathrm{k} 524439.59 / 8016860.74$ & 626 & Rio Verde \\
\hline 2 & RR Soybean & Millet & 5 & $22 \mathrm{k} 482278.90 / 8083495.89$ & 874 & Montividiu \\
\hline 3 & RR Soybean & Sorghum & 1 & 22 k 532499.20/8030623.97 & 685 & Santa Helena \\
\hline 3 & RR Soybean & Sorghum & 2 & $22 \mathrm{k} 525340.12 / 8020605.52$ & 629 & Rio Verde \\
\hline 3 & RR Soybean & Sorghum & 3 & $22 \mathrm{k} \mathrm{480231.77/8099772.54}$ & 847 & Montividiu \\
\hline 3 & RR Soybean & Sorghum & 4 & $22 \mathrm{k} 503766.73 / 8078668.74$ & 792 & Montividiu \\
\hline 3 & RR Soybean & Sorghum & 5 & $22 \mathrm{k} 560464.02 / 8042437.67$ & 530 & Santa Helena \\
\hline 4 & RR Soybean & Fallow & 1 & $22 \mathrm{k} 502135.51 / 8079836.23$ & 756 & Rio Verde \\
\hline 4 & RR Soybean & Fallow & 2 & 22 k 503231.39/8080329.71 & 768 & Rio Verde \\
\hline 4 & RR Soybean & Fallow & 3 & $22 \mathrm{k} 481486.73 / 8099463.40$ & 858 & Montividiu \\
\hline 4 & RR Soybean & Fallow & 4 & $22 \mathrm{k} 480977.40 / 8099669.14$ & 863 & Montividiu \\
\hline 4 & RR Soybean & Fallow & 5 & $22 \mathrm{k} 506231.98 / 8044023.95$ & 832 & Rio Verde \\
\hline 5 & CV Soybean & RR Maize & 1 & $22 \mathrm{k} 500502.47 / 8079165.26$ & 753 & Montividiu \\
\hline 5 & CV Soybean & RR Maize & 2 & 22 k 500701.46/8079859.67 & 750 & Montividiu \\
\hline 5 & CV Soybean & RR Maize & 3 & $22 \mathrm{k} 526129.0 / 8018108.76$ & 666 & Rio Verde \\
\hline 5 & CV Soybean & RR Maize & 4 & 22 k 525957.64/8018272.84 & 660 & Rio Verde \\
\hline 5 & CV Soybean & RR Maize & 5 & $22 \mathrm{k} 518887.66 / 8019237.99$ & 650 & Rio Verde \\
\hline 6 & CV Soybean & Millet & 1 & $22 \mathrm{k} 525933.31 / 8018614.52$ & 654 & Rio Verde \\
\hline 6 & CV Soybean & Millet & 2 & $22 \mathrm{k} 526285.71 / 8018965.53$ & 653 & Rio Verde \\
\hline 6 & CV Soybean & Millet & 3 & 22 k 540987.37/8057939.34 & 569 & St. Antônio da Barra \\
\hline 6 & CV Soybean & Millet & 4 & $22 \mathrm{k} 541670.84 / 8058093.42$ & 574 & St. Antônio da Barra \\
\hline 6 & CV Soybean & Millet & 5 & $22 \mathrm{k} 541911.35 / 8057517.36$ & 584 & St. Antônio da Barra \\
\hline 7 & CV Soybean & Sorghum & 1 & $22 \mathrm{k} 525763.47 / 8018402.43$ & 651 & Rio Verde \\
\hline 7 & CV Soybean & Sorghum & 2 & 22 k 540645.54/8073577.33 & 608 & St. Antônio da Barra \\
\hline 7 & CV Soybean & Sorghum & 3 & $22 \mathrm{k} 540331.35 / 8073928.66$ & 617 & St. Antônio da Barra \\
\hline 7 & CV Soybean & Sorghum & 4 & $22 \mathrm{k} 507115.76 / 8044911.38$ & 779 & Rio Verde \\
\hline 7 & CV Soybean & Sorghum & 5 & 22 k 506929.16/8044116.21 & 819 & Rio Verde \\
\hline
\end{tabular}

RR soybeans (glyphosate-tolerant soybeans) CV soybeans (conventional soybeans).

prior to the application of postemergence herbicide, at 20 days after the implementation of late harvest, or in a fallow area. Weeds were inventoried from the random release of hollow frames in sample areas, and phytosociological analysis was based on the method by Braun-Blanquet (1979). The hollow frames were made of $0.5 \mathrm{~m}$ PVC, which acted as sample units.
Considering the three seasons of the field survey in each agricultural area of 20 ha with five replications, 20 sample units $\left(5 \mathrm{~m}^{2} /\right.$ season or $15 \mathrm{~m}^{2}$ in total) were standardized, amounting to 100 units per treatment $\left(25 \mathrm{~m}^{2} /\right.$ season or $75 \mathrm{~m}^{2}$ in total $)$ and 700 sampling units in each stage of the survey $\left(175 \mathrm{~m}^{2} /\right.$ season or $525 \mathrm{~m}^{2}$ in total), in 2,100 sampled frames-inventories. 
Weeds present in the frames were cut close to the ground and taken to a laboratory for identification and accounting of the number of individuals per species. After botanical identification, they were placed in paper bags to determine the shoot dry matter by drying in a forced ventilation oven at $65{ }^{\circ} \mathrm{C}$ for 72 hours and weighed on a precision scale.

Descriptive analysis of the species found in the areas was conducted through grouping the structural data to calculate the general phytosociological parameters of the study. The importance value index was considered as the main parameter for discussions about severity and species occurrence.

From the quantification of weeds by species and determination of dry biomass, one proceeded to the descriptive analysis, calculating the absolute and relative values of density ( $\mathrm{D}$ and RDe), frequency (F, RF), dominance (ADo and RDo), and importance value index (IVI), following the same methods used by Adegas et al. (2010) and Corrêia et al. (2011), according to the formulas proposed by Mueller-Dombois and Ellenberg (1974), represented in the expressions below:

$\mathrm{D}=$ total number of individuals of the species/total area $\left(\mathrm{m}^{2}\right)$ sampled;

$\mathrm{RDe}=$ species density/density of all species x 100;

$\mathrm{F}=$ number of frames where the species was found/total number of frames of the sampling;

$\mathrm{RF}=$ species frequency/frequency of all species x 100;

$\mathrm{ADo}=$ accumulated dry biomass of the species / total of dry biomass of the sample;

$\mathrm{RDo}=$ dominance of the species/ dominance of all species $x 100$; and

$\mathrm{IVI}=\mathrm{RF}+\mathrm{RDe}+\mathrm{RDo}$.

In the results were presented only the relative values of these indexes. The determination of the floristic composition was done from the phytosociological survey. The Shannon-Wiener ( $\left.H^{\prime}\right)$ diversity indexes, Simpson (D) diversity and equitability (E') for number of individuals were calculated with software PC-ORD 6.1 (McCune \& Mefford, 2011), according to the expressions below:

$$
\begin{aligned}
& \mathrm{S}=\text { richness, } \\
& \mathrm{E}=\mathrm{H} / \ln (\mathrm{S}), \\
& \mathrm{H}=-\Sigma\left(\mathrm{Pi}^{*} \ln (\mathrm{Pi}),\right. \\
& \mathrm{D}=1-\Sigma\left(\mathrm{Pi}{ }^{*} \mathrm{Pi}\right),
\end{aligned}
$$

where: $\mathrm{Pi}=$ probability of importance of the $\mathrm{i}$ species (i species relativized by the total of species in the sample).

Floristic comparison of the relevant species was also held among the different treatments by using the Sorensen (1972) coefficient of similarity, according to the following expression:

$$
\mathrm{SDI}=2 \mathrm{C} /(\mathrm{A}+\mathrm{B})
$$

where: SDI $=$ Sørensen-Dice similarity index; $\mathrm{A}=$ no. of species of area $1 ; \mathrm{B}=$ no. of species of area 2 ; and $\mathrm{C}=$ no. of species common to areas 1 and 2.

\section{RESULTS AND DISCUSSION}

\section{Phytosociological survey}

In the phytosociological survey, the families that had the highest number of collected weeds, in descending order, were: Poaceae, Asteraceae, Euphorbiaceae, Fabaceae, Commelinaceae, Amaranthaceae, Convolvulaceae, Malvaceae, Cyperaceae and Caesalpiniaceae (Table 2). These ten families corresponded to $96 \%$ of the number of individuals. These same families, except for Caesalpiniaceae, were also the most representative in importance value. Regarding the weight in shoot dry matter, which expresses the dominance of each family or species within the community, the highlights, in descending order, were families Poaceae, Asteraceae, Amaranthaceae, Malvaceae, Euphorbiaceae, Commelinaceae, Fabaceae, Smilacaceae, Menispermaceae, and Cyperaceae.

As for the species, Cenchrus echinatus had the greatest number of individuals, dominance and importance value, followed by voluntary or harvested soybeans (Table 3). Because of the importance of the second crop 
Weed phytosociological and floristic survey in agricultural ...

Table 2 - Families phytosociological parameters: dry matter (DM), number of individuals (NI), number of frames (NF), relative frequency $(\mathrm{RF})$, relative density (RDe), relative dominance (RDo), and importance value index (IVI) in southwestern Goiás

\begin{tabular}{|c|c|c|c|c|c|c|c|c|}
\hline Familie & $\mathrm{DM}(\mathrm{g})$ & NI & $\mathrm{NF}$ & $\mathrm{RF}$ & $\mathrm{RDe}$ & RDo & IVI & IVI (\%) \\
\hline Poaceae & $2,336.81$ & 1001 & 950 & 31.64 & 31.10 & 40.18 & 102.92 & 34.31 \\
\hline Asteraceae & $1,495.01$ & 560 & 504 & 16.78 & 17.40 & 25.71 & 59.89 & 19.96 \\
\hline Euphorbiaceae & 197.66 & 377 & 338 & 11.26 & 11.71 & 3.40 & 26.37 & 8.79 \\
\hline Fabaceae & 169.70 & 310 & 308 & 10.26 & 9.63 & 2.92 & 22.81 & 7.60 \\
\hline Amaranthaceae & 401.20 & 257 & 230 & 7.66 & 7.99 & 6.90 & 22.54 & 7.51 \\
\hline Commelinaceae & 193.92 & 261 & 250 & 8.33 & 8.11 & 3.33 & 19.77 & 6.59 \\
\hline Malvaceae & 311.98 & 103 & 98 & 3.26 & 3.20 & 5.36 & 11.83 & 3.94 \\
\hline Convolvulaceae & 75.49 & 111 & 110 & 3.66 & 3.45 & 1.30 & 8.41 & 2.80 \\
\hline Cyperaceae & 80.26 & 86 & 85 & 2.83 & 2.67 & 1.38 & 6.88 & 2.29 \\
\hline Smilacaceae & 152.36 & 18 & 14 & 0.47 & 0.56 & 2.62 & 3.65 & 1.22 \\
\hline Menispermaceae & 104.09 & 27 & 24 & 0.80 & 0.84 & 1.79 & 3.43 & 1.14 \\
\hline Caesalpiniaceae & 73.55 & 34 & 29 & 0.97 & 1.06 & 1.26 & 3.29 & 1.10 \\
\hline Rubiaceae & 49.13 & 24 & 23 & 0.77 & 0.75 & 0.84 & 2.36 & 0.79 \\
\hline Polygonaceae & 31.84 & 8 & 8 & 0.27 & 0.25 & 0.55 & 1.06 & 0.35 \\
\hline Lamiaceae & 16.16 & 12 & 10 & 0.33 & 0.37 & 0.28 & 0.98 & 0.33 \\
\hline Mimosaceae & 35.11 & 5 & 5 & 0.17 & 0.16 & 0.60 & 0.93 & 0.31 \\
\hline Myrtaceae & 22.93 & 5 & 5 & 0.17 & 0.16 & 0.39 & 0.72 & 0.24 \\
\hline Solanaceae & 19.42 & 5 & 5 & 0.17 & 0.16 & 0.33 & 0.66 & 0.22 \\
\hline Boraginaceae & 16.51 & 1 & 1 & 0.03 & 0.03 & 0.28 & 0.35 & 0.12 \\
\hline Malpighiaceae & 7.29 & 3 & 2 & 0.07 & 0.09 & 0.13 & 0.29 & 0.10 \\
\hline Chrysobalanaceae & 7.63 & 1 & 1 & 0.03 & 0.03 & 0.13 & 0.20 & 0.07 \\
\hline Nyctagynaceae & 3.83 & 2 & 2 & 0.07 & 0.06 & 0.07 & 0.19 & 0.06 \\
\hline Simaroubaceae & 6.72 & 1 & 1 & 0.03 & 0.03 & 0.12 & 0.18 & 0.06 \\
\hline Phyllanthaceae & 0.93 & 2 & 2 & 0.07 & 0.06 & 0.02 & 0.14 & 0.05 \\
\hline Anacardiaceae & 0.90 & 2 & 2 & 0.07 & 0.06 & 0.02 & 0.14 & 0.05 \\
\hline Moraceae & 4.03 & 1 & 1 & 0.03 & 0.03 & 0.07 & 0.13 & 0.04 \\
\hline Connaraceae & 0.85 & 1 & 1 & 0.03 & 0.03 & 0.01 & 0.08 & 0.03 \\
\hline Vochysiaceae & 0.45 & 1 & 1 & 0.03 & 0.03 & 0.01 & 0.07 & 0.02 \\
\hline Total & $5,815.74$ & 3,219 & 2,100 & 100 & 100 & 100 & 300 & 100 \\
\hline
\end{tabular}

in this region, resurgent soybeans, known as harvested, were classified as invasive of late harvest. The harvested ones were recorded with high frequency in crop rotation. This situation was observed in all the studied areas, demonstrating grain losses in mechanized harvesting and competition with the second harvest crops. Furthermore, the presence of soybeans in agricultural areas throughout the period of fallowing favors the development of phytosanitary problems. Other eight species (Chamaesyce hirta, Commelina benghalensis, Alternanthera tenella, Bidens subalternans, Sida glaziovii, Eleusine indica, Euphorbia heterophylla, and Cyperus difformis) were among the ten most significant in importance values.

As far dominance as is concerned, after C. echinatus, stood out: Conyza bonariensis, A. tenella, S. glaziovii, Praxelis pauciflora, C. benghalensis, Malvastrum coromandelianum, Conyza canadensis, C. hirta and E. indica.

In areas of rotated crops in the cerrado of Roraima, 23 species were recorded (Cruz et al., 2009), of which nine (E. heterophylla, Urochloa decumbens, C. benghalensis, A. tenella, S. glaziovii, S. obtusifolia, E. indica, C. hyrta and Portulaca oleraceae) were common to this survey. Of these species, six are among 
Table 3 - Species phytosociological parameters: dry matter (DM), number of individuals (NI), number of frames (NF), relative frequency $(\mathrm{RF})$, relative density (RDe), relative dominance (RDo), and importance value index (IVI) in southwestern Goiás

\begin{tabular}{|c|c|c|c|c|c|c|c|c|}
\hline Species & $\mathrm{DM}(\mathrm{g})$ & $\mathrm{NI}$ & NF & RF & $\mathrm{RDe}$ & RDo & IVI & IVI $(\%)$ \\
\hline Cenchrus echinatus & $1,589.75$ & 680 & 640 & 21.312 & 21.125 & 27.34 & 69.77 & 23.257 \\
\hline Alternanthera tenella & 367.41 & 244 & 217 & 7.211 & 7.580 & 6.32 & 21.11 & 7.036 \\
\hline Commelina benghalensis & 193.92 & 261 & 250 & 8.308 & 8.108 & 3.33 & 19.75 & 6.583 \\
\hline Glycine max & 85.89 & 284 & 283 & 9.404 & 8.823 & 1.48 & 19.70 & 6.568 \\
\hline Chamaesyce hirta & 146.70 & 276 & 247 & 8.208 & 8.574 & 2.52 & 19.30 & 6.435 \\
\hline Sida glaziovii & 366.72 & 134 & 122 & 4.054 & 4.163 & 6.31 & 14.52 & 4.841 \\
\hline Bidens subalternans & 111.68 & 176 & 156 & 5.184 & 5.468 & 1.92 & 12.57 & 4.191 \\
\hline Eleusine indica & 143.46 & 112 & 117 & 3.888 & 3.479 & 2.47 & 9.83 & 3.278 \\
\hline Conyza bonariensis & 398.78 & 44 & 44 & 1.462 & 1.367 & 6.86 & 9.69 & 3.229 \\
\hline Praxelis pauciflora & 198.82 & 60 & 48 & 1.595 & 1.864 & 3.42 & 6.88 & 2.293 \\
\hline Cyperus difformis & 79.21 & 85 & 84 & 2.791 & 2.641 & 1.36 & 6.79 & 2.265 \\
\hline Euphorbia heterophyla & 35.82 & 100 & 90 & 2.991 & 3.107 & 0.62 & 6.71 & 2.238 \\
\hline Conyza canadensis & 151.66 & 48 & 45 & 1.495 & 1.491 & 2.61 & 5.59 & 1.865 \\
\hline Sida rhombifolia & 136.92 & 48 & 48 & 1.595 & 1.491 & 2.35 & 5.44 & 1.814 \\
\hline Tridax procumbens & 103.21 & 56 & 51 & 1.695 & 1.740 & 1.77 & 5.21 & 1.736 \\
\hline Malvastrum coromandelianum & 152.24 & 39 & 34 & 1.130 & 1.212 & 2.62 & 4.96 & 1.653 \\
\hline Ipomoea grandifolia & 42.00 & 65 & 65 & 2.160 & 2.019 & 0.72 & 4.90 & 1.634 \\
\hline Digitaria horizontalis & 94.55 & 44 & 35 & 1.163 & 1.367 & 1.63 & 4.16 & 1.385 \\
\hline Pennisetum setosum & 74.39 & 45 & 40 & 1.329 & 1.398 & 1.28 & 4.01 & 1.335 \\
\hline Ipomoea cordifolia & 33.49 & 46 & 45 & 1.495 & 1.429 & 0.58 & 3.50 & 1.167 \\
\hline Panicum maximum & 138.99 & 13 & 13 & 0.432 & 0.404 & 2.39 & 3.23 & 1.075 \\
\hline Senna obtusifolia & 73.24 & 33 & 28 & 0.930 & 1.025 & 1.26 & 3.21 & 1.072 \\
\hline Setaria parviflora & 91.71 & 22 & 21 & 0.698 & 0.683 & 1.58 & 2.96 & 0.986 \\
\hline Gnaphalium coarctatum & 43.29 & 22 & 20 & 0.665 & 0.683 & 0.74 & 2.09 & 0.697 \\
\hline Smilax polyantha & 86.61 & 9 & 7 & 0.233 & 0.280 & 1.49 & 2.00 & 0.667 \\
\hline Rhinchelytrum repens & 49.93 & 15 & 15 & 0.498 & 0.466 & 0.86 & 1.82 & 0.608 \\
\hline Digitaria insularis & 26.81 & 21 & 21 & 0.698 & 0.652 & 0.46 & 1.81 & 0.604 \\
\hline Crotalaria spectabilis & 40.78 & 15 & 14 & 0.465 & 0.466 & 0.70 & 1.63 & 0.544 \\
\hline Cissampelos sp2. & 60.95 & 10 & 8 & 0.266 & 0.311 & 1.05 & 1.62 & 0.541 \\
\hline Richardia brasiliense & 37.15 & 14 & 13 & 0.432 & 0.435 & 0.64 & 1.51 & 0.502 \\
\hline Zea mays & 24.07 & 16 & 16 & 0.532 & 0.497 & 0.41 & 1.44 & 0.481 \\
\hline Amaranthus viridis & 33.79 & 13 & 13 & 0.432 & 0.404 & 0.58 & 1.42 & 0.472 \\
\hline Brachiaria sp. & 35.19 & 11 & 11 & 0.366 & 0.342 & 0.61 & 1.31 & 0.437 \\
\hline Emilia fosbergii & 47.97 & 6 & 6 & 0.199 & 0.186 & 0.82 & 1.21 & 0.404 \\
\hline Pennisetum americanum & 10.44 & 16 & 16 & 0.532 & 0.497 & 0.18 & 1.21 & 0.403 \\
\hline Cissampelos ovolifolia & 28.41 & 8 & 8 & 0.266 & 0.249 & 0.49 & 1.00 & 0.334 \\
\hline Leonotis nepetaefolia & 15.00 & 12 & 10 & 0.332 & 0.373 & 0.26 & 0.96 & 0.321 \\
\hline Smilax brasiliensis & 39.41 & 5 & 3 & 0.100 & 0.155 & 0.68 & 0.93 & 0.311 \\
\hline Mimosa hirsutissima & 35.11 & 5 & 5 & 0.166 & 0.155 & 0.60 & 0.93 & 0.308 \\
\hline Cissampelos sp1. & 14.73 & 9 & 8 & 0.266 & 0.280 & 0.25 & 0.80 & 0.266 \\
\hline Andira vermifuga & 30.74 & 4 & 4 & 0.133 & 0.124 & 0.53 & 0.79 & 0.262 \\
\hline Sorghum halepense & 38.00 & 1 & 1 & 0.033 & 0.031 & 0.65 & 0.72 & 0.239 \\
\hline Solanum americanum & 19.42 & 5 & 5 & 0.166 & 0.155 & 0.33 & 0.66 & 0.218 \\
\hline
\end{tabular}

To be continued... 
Tabela 3, cont.

\begin{tabular}{|c|c|c|c|c|c|c|c|c|}
\hline Species & $\mathrm{DM}(\mathrm{g})$ & $\mathrm{NI}$ & NF & RF & $\mathrm{RDe}$ & RDo & IVI & IVI $(\%)$ \\
\hline$\overline{\text { Digitaria ciliares }}$ & 19.51 & 5 & 4 & 0.133 & 0.155 & 0.34 & 0.62 & 0.208 \\
\hline Vernonia ferruginea & 28.20 & 2 & 2 & 0.066 & 0.062 & 0.48 & 0.61 & 0.204 \\
\hline Spermacoce latifolia & 7.19 & 7 & 7 & 0.233 & 0.217 & 0.12 & 0.57 & 0.191 \\
\hline Smilax campestris & 21.65 & 3 & 3 & 0.100 & 0.093 & 0.37 & 0.57 & 0.188 \\
\hline Rumex acetoselha & 9.38 & 6 & 6 & 0.199 & 0.186 & 0.16 & 0.55 & 0.182 \\
\hline Sida cordifolia & 12.45 & 5 & 5 & 0.166 & 0.155 & 0.21 & 0.54 & 0.178 \\
\hline Rumex obtusifolius L. & 22.46 & 2 & 2 & 0.066 & 0.062 & 0.39 & 0.51 & 0.172 \\
\hline Bidens pilosa & 11.04 & 6 & 4 & 0.133 & 0.186 & 0.19 & 0.51 & 0.170 \\
\hline Indigofera hirsuta & 5.61 & 6 & 6 & 0.199 & 0.186 & 0.10 & 0.48 & 0.161 \\
\hline Eugenia sp. & 15.03 & 3 & 3 & 0.100 & 0.093 & 0.26 & 0.45 & 0.150 \\
\hline Acanthospermum hispidum & 18.65 & 2 & 2 & 0.066 & 0.062 & 0.32 & 0.45 & 0.150 \\
\hline Synedrellopsis grisebachii & 12.41 & 3 & 3 & 0.100 & 0.093 & 0.21 & 0.41 & 0.135 \\
\hline Heliotropium indicum & 16.51 & 1 & 1 & 0.033 & 0.031 & 0.28 & 0.35 & 0.116 \\
\hline Cnidoscolus urens & 15.13 & 1 & 1 & 0.033 & 0.031 & 0.26 & 0.32 & 0.108 \\
\hline Sida urens & 7.19 & 3 & 3 & 0.100 & 0.093 & 0.12 & 0.32 & 0.106 \\
\hline Heteropterys sp. & 7.29 & 3 & 2 & 0.066 & 0.093 & 0.13 & 0.29 & 0.095 \\
\hline Spermacoce verticilata & 4.79 & 3 & 3 & 0.100 & 0.093 & 0.08 & 0.28 & 0.092 \\
\hline Myrcia guianensis & 7.90 & 2 & 2 & 0.066 & 0.062 & 0.14 & 0.26 & 0.088 \\
\hline Pavonia rosa-campestris & 1.24 & 3 & 3 & 0.100 & 0.093 & 0.02 & 0.21 & 0.071 \\
\hline Gossypum hirsutum & 0.19 & 3 & 3 & 0.100 & 0.093 & 0.00 & 0.20 & 0.065 \\
\hline Couepia grandiflora & 7.63 & 1 & 1 & 0.033 & 0.031 & 0.13 & 0.20 & 0.065 \\
\hline Neea theifera & 3.83 & 2 & 2 & 0.066 & 0.062 & 0.07 & 0.19 & 0.065 \\
\hline Simaba sp. & 6.72 & 1 & 1 & 0.033 & 0.031 & 0.12 & 0.18 & 0.060 \\
\hline Crotalaria incana & 6.68 & 1 & 1 & 0.033 & 0.031 & 0.11 & 0.18 & 0.060 \\
\hline Smilax ovolifolia & 4.69 & 1 & 1 & 0.033 & 0.031 & 0.08 & 0.14 & 0.048 \\
\hline Phyllanthus tenellus & 0.93 & 2 & 2 & 0.066 & 0.062 & 0.02 & 0.14 & 0.048 \\
\hline Brosimum gaudichaudii & 4.03 & 1 & 1 & 0.033 & 0.031 & 0.07 & 0.13 & 0.045 \\
\hline Sida spinosa & 1.75 & 1 & 1 & 0.033 & 0.031 & 0.03 & 0.09 & 0.031 \\
\hline Ageratum conyzoides & 1.40 & 1 & 1 & 0.033 & 0.031 & 0.02 & 0.09 & 0.029 \\
\hline Cresta sphaerocephala & 1.20 & 1 & 1 & 0.033 & 0.031 & 0.02 & 0.08 & 0.028 \\
\hline Hyptis lophanta & 1.16 & 1 & 1 & 0.033 & 0.031 & 0.02 & 0.08 & 0.028 \\
\hline Cyperus odoratus & 1.05 & 1 & 1 & 0.033 & 0.031 & 0.02 & 0.08 & 0.027 \\
\hline Lithraea molleoides & 0.90 & 1 & 1 & 0.033 & 0.031 & 0.02 & 0.08 & 0.027 \\
\hline Connarus suberosus & 0.85 & 1 & 1 & 0.033 & 0.031 & 0.01 & 0.08 & 0.026 \\
\hline Qualea parviflora & 0.45 & 1 & 1 & 0.033 & 0.031 & 0.01 & 0.07 & 0.024 \\
\hline Bauhinia sp. & 0.31 & 1 & 1 & 0.033 & 0.031 & 0.01 & 0.07 & 0.023 \\
\hline TOTAL & $5,815.74$ & 3,219 & 2,100 & 100 & 100 & 100 & 300 & 100 \\
\hline
\end{tabular}

sp1: species 1 of genus Cissampelos; sp2: species 2 of genus Cissampelos.

the greatest number of individuals and importance value in this study, excluding only $S$. obtusifolia and P. oleracea. Foltran et al. (2010), by means of a phytosociological survey in crop rotation in the Brazilian city of Botucatu, SP, have recorded the occurrence of species A. tenella and C. benghalensis in the crop-fallow system and green manure crop even with low density. Both species are among the ten ones with the highest importance value for this study. Adegas et al. (2010), in sunflower cultivation, have found that C. hirta, $E$. heterophyla, C. benghalensis, C. echinatus, and A. tenella had higher levels of importance for Brazilian states of Goiás and Mato Grosso do Sul - data similar to the ones in the present study. 
In qualitative and quantitative assessments in soybean production areas of conventional direct seeding, Pereira et al. (2000) have recorded in Botucatu, SP, eight species similar to this ones in this survey, as follows: Sida rhombifolia, C. benghalensis, E. heterophylla, Amaranthus viridis, Acanthospermum hispidum, Digitaria horizontalis, C. echinatus and Bidens pilosa. These floristic similarities in relation to the importance values for different regions of the country demonstrate predominance of some species of weeds in soybeans crops. This may be associated with tolerance or resistance to the main herbicides used on this crop.

\section{Floristic composition}

As for the floristic composition, 79 species were recorded, belonging to 28 families and 58 botanical genera (Tables 4 and 5). The distribution by classes consisted of 59 species,

Table 4 - Floristic listing of magnoliopsida weed species recorded in southwestern Goiás

\begin{tabular}{|c|c|c|c|c|c|c|c|c|c|}
\hline \multirow[b]{2}{*}{$\begin{array}{l}\text { Families/class: } \\
\text { Magnoliopsida }\end{array}$} & \multirow[b]{2}{*}{ Species } & \multirow[b]{2}{*}{ Common name } & \multicolumn{7}{|c|}{ Treatment } \\
\hline & & & 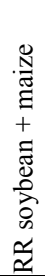 & 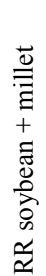 & 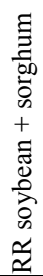 & 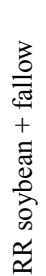 & 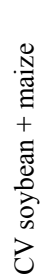 & 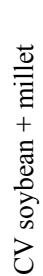 & 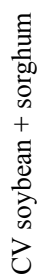 \\
\hline \multirow[b]{2}{*}{ 1. Amaranthaceae } & Alternanthera tenella Colla & Joyweeds & $\mathrm{X}$ & $\mathrm{X}$ & $\mathrm{X}$ & $\mathrm{X}$ & $\mathrm{X}$ & $\mathrm{X}$ & $\mathrm{X}$ \\
\hline & Amaranthus viridis $\mathrm{L}$. & $\begin{array}{l}\text { Large-fruit amaranth, } \\
\text { low amaranth, or } \\
\text { Argentina amaranth. }\end{array}$ & $\mathrm{X}$ & & $\mathrm{X}$ & $\mathrm{X}$ & $\mathrm{X}$ & & $\mathrm{X}$ \\
\hline 2. Anacardiaceae & Lithraea molleoides (Vell.) Engl. & Aroeira-branca & $\mathrm{X}$ & & & & & & \\
\hline \multirow{13}{*}{ 3. Asteraceae } & Acanthospermum hispidum DC. & $\begin{array}{l}\text { Bristly starbur, goat's } \\
\text { head, hispid starburr, or } \\
\text { starbur }\end{array}$ & & $\mathrm{X}$ & & & & & \\
\hline & Ageratum conyzoides L. & $\begin{array}{l}\text { Billygoat-weed, chick } \\
\text { weed, goatweed, } \\
\text { whiteweed }\end{array}$ & & & & $\mathrm{X}$ & & & \\
\hline & Bidens subalternans DC. & $\begin{array}{l}\text { Beggarticks, black jack, } \\
\text { burr marigolds, } \\
\text { cobbler's pegs, Spanish } \\
\text { needles, stickseeds, } \\
\text { tickseeds, or tickseed } \\
\text { sunflowers }\end{array}$ & $X$ & $\mathrm{X}$ & $\mathrm{X}$ & $X$ & $\mathrm{X}$ & $\mathrm{X}$ & $\mathrm{X}$ \\
\hline & Bidens pilosa $\mathrm{L}$. & $\begin{array}{l}\text { Black-jack, } \\
\text { beggarticks, cobbler's } \\
\text { pegs, or Spanish } \\
\text { needle. }\end{array}$ & $X$ & $\mathrm{X}$ & & & & & \\
\hline & Conyza bonariensis (L.) Cronquist & Horseweed & $\mathrm{X}$ & $\mathrm{X}$ & $\mathrm{X}$ & $\mathrm{X}$ & & $\mathrm{X}$ & $\mathrm{X}$ \\
\hline & Conyza canadensis (L.) Cronquist & Canadian horseweed & $\mathrm{X}$ & $\mathrm{X}$ & & $\mathrm{X}$ & $\mathrm{X}$ & $\mathrm{X}$ & \\
\hline & Cresta sphaerocephala DC. & João-bobo & & & $\mathrm{X}$ & & & & \\
\hline & Emilia fosbergii Nicolson & Florida tasselflower. & & & & $\mathrm{X}$ & & $\mathrm{X}$ & \\
\hline & Gnaphalium coarctatum Willd. & Cudweed & $\mathrm{X}$ & & $\mathrm{X}$ & $\mathrm{X}$ & $\mathrm{X}$ & $\mathrm{X}$ & \\
\hline & $\begin{array}{l}\text { Praxelis pauciflora (Kunth) R. M. } \\
\text { King e H. Rob. }\end{array}$ & Anil & $X$ & $\mathrm{X}$ & $\mathrm{X}$ & $\mathrm{X}$ & $\mathrm{X}$ & $\mathrm{X}$ & \\
\hline & $\begin{array}{l}\text { Synedrellopsis grisebachii Hieron \& } \\
\text { Kuntze }\end{array}$ & Straggler daisy & & $\mathrm{X}$ & & $X$ & & & \\
\hline & Tridax procumbens $\mathrm{L}$. & $\begin{array}{l}\text { Coat buttons or tridax } \\
\text { daisy }\end{array}$ & $X$ & $\mathrm{X}$ & $\mathrm{X}$ & $\mathrm{X}$ & $\mathrm{X}$ & $X$ & $\mathrm{X}$ \\
\hline & Vernonia ferruginea Less. & Ironweed & & $\mathrm{X}$ & & & & & \\
\hline
\end{tabular}

To be continued.. 
Tabela 4, cont.

\begin{tabular}{|c|c|c|c|c|c|c|c|c|c|}
\hline \multirow[b]{2}{*}{$\begin{array}{l}\text { Families/class: } \\
\text { Magnoliopsida }\end{array}$} & \multirow[b]{2}{*}{ Species } & \multirow[b]{2}{*}{ Common name } & \multicolumn{7}{|c|}{ Treatment } \\
\hline & & & 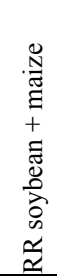 & 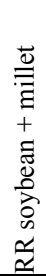 & 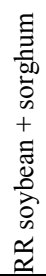 & 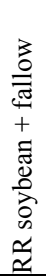 & 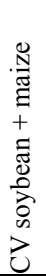 & 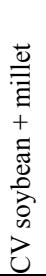 & 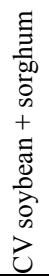 \\
\hline 4. Boraginaceae & Heliotropium indicum $\mathrm{L}$. & Indian heliotrope & & $\mathrm{X}$ & & & & & \\
\hline \multirow[t]{2}{*}{ 5. Caesalpiniaceae } & $\begin{array}{l}\text { Senna obtusifolia (L.) H. S. Irwin e } \\
\text { Barneby }\end{array}$ & $\begin{array}{l}\text { Chinese Senna or } \\
\text { Sicklepod. } \\
\end{array}$ & $\mathrm{X}$ & $\mathrm{X}$ & $\mathrm{X}$ & $X$ & $\mathrm{X}$ & $\mathrm{X}$ & $\mathrm{X}$ \\
\hline & Bauhinia sp. & Orchid tree & $\mathrm{X}$ & & & & & & \\
\hline 6. Chrysobalanaceae & Couepia grandiflora Benth. & Oiti & & & $\mathrm{X}$ & & & & \\
\hline 7. Connaraceae & Connarus suberosus L. & Pau-de-brinco & $\mathrm{X}$ & & & & & & \\
\hline \multirow[b]{2}{*}{ 8. Convolvulaceae } & Ipomoea cordifolia L. (triloba) & $\begin{array}{l}\text { Heart-leaved morning } \\
\text { glory }\end{array}$ & $\mathrm{X}$ & $\mathrm{X}$ & & $X$ & $X$ & $\mathrm{X}$ & \\
\hline & Ipomoea grandifolia $\mathrm{L}$. & $\begin{array}{l}\text { Morning glory, sweet } \\
\text { potato, bindweed, } \\
\text { moonflower, etc. }\end{array}$ & $\mathrm{X}$ & $\mathrm{X}$ & $\mathrm{X}$ & $X$ & $\mathrm{X}$ & $\mathrm{X}$ & $\mathrm{X}$ \\
\hline \multirow[t]{3}{*}{ 9. Euphorbiaceae } & Chamaesyce hirta (L.) Millsp. & $\begin{array}{l}\text { Asthma plant, asthma } \\
\text { weed, asthmaplant, cat's } \\
\text { hair, flowery headed } \\
\text { spurge, garden spurge, } \\
\text { hairy spurge, Jean } \\
\text { Roberts, old blood, pill } \\
\text { bearing spurge, pill- } \\
\text { bearing spurge, pillpod } \\
\text { sandmat, pillpod spurge, } \\
\text { Queensland asthma } \\
\text { weed, red euphorbia, } \\
\text { red milkweed, snake } \\
\text { weed, snakeweed, } \\
\text { sneeze weed, or spurge } \\
\end{array}$ & $\mathrm{X}$ & $\mathrm{X}$ & $\mathrm{X}$ & $X$ & $X$ & $\mathrm{X}$ & $X$ \\
\hline & Cnidoscolus urens (L.) Arthur & $\begin{array}{l}\text { 'Bull nettle', 'spurge } \\
\text { nettle', or 'mala mujer' } \\
\text { (evil woman). }\end{array}$ & & & & & & $\mathrm{X}$ & \\
\hline & Euphorbia heterophyla L. & $\begin{array}{l}\text { (Mexican) fireplant, } \\
\text { painted euphorbia, } \\
\text { Japanese poinsettia, } \\
\text { desert poinsettia, wild } \\
\text { poinsettia, fire on the } \\
\text { mountain, paintedleaf, } \\
\text { painted spurge, } \\
\text { milkweed, and kaliko } \\
\text { plant }\end{array}$ & $X$ & $\mathrm{X}$ & $\mathrm{X}$ & $X$ & $\mathrm{X}$ & $\mathrm{X}$ & $\mathrm{X}$ \\
\hline \multirow{5}{*}{ 10. Fabaceae } & Andira vermifuga Mart. Ex Benth. & Angelim-do-cerrado & $\mathrm{X}$ & & & $\mathrm{X}$ & $\mathrm{X}$ & & \\
\hline & Crotalaria incana $\mathrm{L}$. & Woolly rattlepod & & $\mathrm{X}$ & & & & & \\
\hline & Crotalaria spectabilis Roth & Rattlepod or rattlebox & $\mathrm{X}$ & & & & & & \\
\hline & Glycine $\max \mathrm{L}$. & Soybean & $\mathrm{X}$ & $\mathrm{X}$ & $\mathrm{X}$ & $\mathrm{X}$ & $\mathrm{X}$ & $\mathrm{X}$ & $\mathrm{X}$ \\
\hline & Indigofera hirsuta $\mathrm{L}$. & $\begin{array}{l}\text { Hairy indigo, rough } \\
\text { hairy indigo }\end{array}$ & $\mathrm{X}$ & & & & & & \\
\hline \multirow{4}{*}{ 11. Lamiaceae } & Leonotis nepetifolia (L.) R. Br. & $\begin{array}{l}\text { Klip dagga, Christmas } \\
\text { candlestick, or lion's ear }\end{array}$ & & $\mathrm{X}$ & $\mathrm{X}$ & & $X$ & $\mathrm{X}$ & \\
\hline & Hyptis lophantha Mart. Ex Benth & Bushmint & & & & $\mathrm{X}$ & & & \\
\hline & Mimosa hirsutissima Mart. & Malícia & $\mathrm{X}$ & $\mathrm{X}$ & & & & $\mathrm{X}$ & \\
\hline & Heteropterys sp. & ------- & & $\mathrm{X}$ & $\mathrm{X}$ & & & & \\
\hline
\end{tabular}

To be continued.. 
Tabela 4, cont.

\begin{tabular}{|c|c|c|c|c|c|c|c|c|c|}
\hline \multirow{2}{*}{$\begin{array}{l}\text { Families/class: } \\
\text { Magnoliopsida }\end{array}$} & \multirow[b]{2}{*}{ Species } & \multirow[b]{2}{*}{ Common name } & \multicolumn{7}{|c|}{ Treatment } \\
\hline & & & 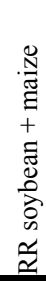 & 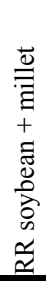 & 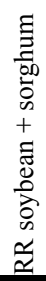 & 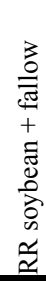 & 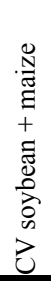 & 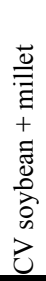 & 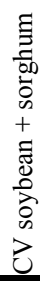 \\
\hline \multirow{8}{*}{ 14. Malvaceae } & Gossypum hirsutum L. & $\begin{array}{l}\text { Upland cotton or Mexican } \\
\text { cotton }\end{array}$ & $\mathrm{X}$ & & & & & & \\
\hline & $\begin{array}{l}\text { Malvastrum coromandelianum (L.) } \\
\text { Garcke }\end{array}$ & $\begin{array}{l}\text { False mallow, broom weed, } \\
\text { Clock plant, or prickly } \\
\text { malvastrum }\end{array}$ & $\mathrm{X}$ & $\mathrm{X}$ & $\mathrm{X}$ & $\mathrm{X}$ & & & $X$ \\
\hline & Pavonia rosa-campestris A. St. Hill & Rosa-vermelha & $\mathrm{X}$ & & $\mathrm{X}$ & & & & \\
\hline & Sida cordifolia $\mathrm{L}$. & $\begin{array}{l}\text { Country-mallow, flannel } \\
\text { sida }\end{array}$ & & $\mathrm{X}$ & & & $\mathrm{X}$ & & \\
\hline & Sida glaziovii K. Schum & Malva & $\mathrm{X}$ & $\mathrm{X}$ & $\mathrm{X}$ & $\mathrm{X}$ & $\mathrm{X}$ & $\mathrm{X}$ & $\mathrm{X}$ \\
\hline & Sida spinosa L. & Prickly fanpetals & & & & & $\mathrm{X}$ & & \\
\hline & Sida rhombifolia L. & Paddy's lucerne, jelly leaf & $\mathrm{X}$ & $\mathrm{X}$ & $\mathrm{X}$ & $\mathrm{X}$ & $\mathrm{X}$ & $\mathrm{X}$ & \\
\hline & Sida urens L. & $\begin{array}{l}\text { Tropical fanpetals, balai- } \\
\text { zortie }\end{array}$ & & $\mathrm{X}$ & $\mathrm{X}$ & $\mathrm{X}$ & & & \\
\hline \multirow{3}{*}{ 15. Menispermaceae } & Cissampelos ovolifolia DC. & Orelha-de-onça & $\mathrm{X}$ & $\mathrm{X}$ & $\mathrm{X}$ & & & & \\
\hline & Cissampelos sp2. & Orelha-de-onça & & & $\mathrm{X}$ & & $\mathrm{X}$ & $\mathrm{X}$ & \\
\hline & Cissampelos sp1. & Orelha-de-onça & $\mathrm{X}$ & & $\mathrm{X}$ & & $\mathrm{X}$ & & \\
\hline 16. Moraceae & Brosimum gaudichaudii Trécul & Mama cadela & & & & $\mathrm{X}$ & & & \\
\hline \multirow[b]{2}{*}{ 17. Myrtaceae } & Eugenia sp. & Cagaita & & & $\mathrm{X}$ & & $\mathrm{X}$ & & \\
\hline & Myrcia guianensis (Aubl.) DC. & $\begin{array}{l}\text { Birch, bois de fer, bois de } \\
\text { Ste. Lucie, bois petite } \\
\text { feuille, guava berry }\end{array}$ & & $\mathrm{X}$ & $\mathrm{X}$ & & & & \\
\hline 18. Nyctagynaceae & Neea theifera Oerst. & Nia, neea, or saltwood. & & & & $\mathrm{X}$ & & & \\
\hline 19. Phyllanthaceae & Phyllanthus tenellus Roxb. & Leafflower & $\mathrm{X}$ & & & & & $\mathrm{X}$ & \\
\hline \multirow[b]{2}{*}{ 20. Polygonaceae } & Rumex acetoselha $\mathrm{L}$. & $\begin{array}{l}\text { Sheep's sorrel, red sorrel, } \\
\text { sour weed, or field sorrel }\end{array}$ & & & & $\mathrm{X}$ & $\mathrm{X}$ & & $\mathrm{X}$ \\
\hline & Rumex obtusifolius L. & $\begin{array}{l}\text { Bitter dock, broad-leaved } \\
\text { dock, bluntleaf dock, dock } \\
\text { leaf, or butter dock }\end{array}$ & & $\mathrm{X}$ & & & & & \\
\hline \multirow{3}{*}{ 21. Rubiaceae } & Richardia brasiliensis Gomes & $\begin{array}{l}\text { Tropical Mexican clover, } \\
\text { Brazilian calla-lily, white- } \\
\text { eye, for Brazil pusley }\end{array}$ & & $\mathrm{X}$ & $\mathrm{X}$ & & $\mathrm{X}$ & $\mathrm{X}$ & \\
\hline & Spermacoce latifolia Aubl. & Buttonweed & & & $\mathrm{X}$ & $\mathrm{X}$ & $\mathrm{X}$ & & \\
\hline & Spermacoce verticilata L. & Shrubby false buttonweed & & & $\mathrm{X}$ & & & & \\
\hline 22. Simaroubaceae & Simaba sp. & ------- & & & $\mathrm{X}$ & & & & \\
\hline 23. Solanaceae & Solanum americanum Mill. & $\begin{array}{l}\text { American nightshade or } \\
\text { glossy nightshade }\end{array}$ & & & & $\mathrm{X}$ & $\mathrm{X}$ & & \\
\hline 24. Vochysiaceae & Qualea parviflora Mart. & Pau-terra & $\mathrm{X}$ & & & & & & \\
\hline
\end{tabular}

RR soybeans (glyphosate-tolerant soybeans); CV soybeans (conventional soybeans).

24 families and 45 genera belonging to the Magnoliopsidas (eudicotyledonous), and 20 species, 4 families and 13 genera belonging to the Liliopsidas (monocotyledonous).
Families which showed the greatest number of species were: Asteraceae, Poaceae, Malvaceae, Fabaceae, Smilacaceae, Euphorbiaceae, Menispermaceae and 
Table 5 - Floristic listing of liliopsida weeds species in alphabetical order of families recorded in southwester Goiás

\begin{tabular}{|c|c|c|c|c|c|c|c|c|c|}
\hline \multirow[b]{2}{*}{$\begin{array}{l}\text { Families/class: } \\
\text { Liliopsida }\end{array}$} & \multirow[b]{2}{*}{ Species } & \multirow[b]{2}{*}{ Common name } & \multicolumn{7}{|c|}{ Treatment } \\
\hline & & & 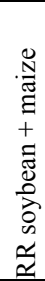 & 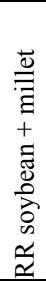 & 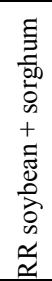 & 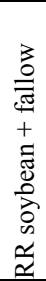 & 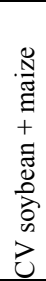 & 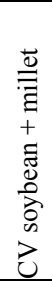 & 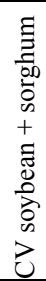 \\
\hline 1. Commelinaceae & Commelina benghalensis L. & Benghal dayflower & $\mathrm{X}$ & $\mathrm{X}$ & $\mathrm{X}$ & $\mathrm{X}$ & $\mathrm{X}$ & $\mathrm{X}$ & $\mathrm{X}$ \\
\hline \multirow[b]{2}{*}{ 2. Cyperaceae } & Cyperus difformis $\mathrm{L}$. & $\begin{array}{l}\text { Variable flatsedge or } \\
\text { smallflower umbrella- } \\
\text { sedge }\end{array}$ & $\mathrm{X}$ & $\mathrm{X}$ & $\mathrm{X}$ & $\mathrm{X}$ & $\mathrm{X}$ & $\mathrm{X}$ & $\mathrm{X}$ \\
\hline & Cyperus odoratus L. & $\begin{array}{l}\text { Cyperus odoratus is a } \\
\text { species of sedge } \\
\text { known by Fragrant } \\
\text { flatsedge or rusty } \\
\text { flatsedge }\end{array}$ & & $\mathrm{X}$ & & & & & \\
\hline \multirow{13}{*}{ 3. Poaceae } & Brachiaria decumbens Stapf. & Signalgrass & $\mathrm{X}$ & $\mathrm{X}$ & & & $\mathrm{X}$ & & \\
\hline & Cenchrus echinatus L. & $\begin{array}{l}\text { Southern sandspur or } \\
\text { southern sandbur }\end{array}$ & $\mathrm{X}$ & $\mathrm{X}$ & $\mathrm{X}$ & $\mathrm{X}$ & $\mathrm{X}$ & $\mathrm{X}$ & $\mathrm{X}$ \\
\hline & Digitaria ciliares (Retz) Koeler & $\begin{array}{l}\text { Southern crabgrass, } \\
\text { tropical crabgrass, or } \\
\text { summer grass. }\end{array}$ & & & & $\mathrm{X}$ & & & \\
\hline & Digitaria horizontalis Willd. & Jamaican crabgrass & $\mathrm{X}$ & $\mathrm{X}$ & $\mathrm{X}$ & $\mathrm{X}$ & & $\mathrm{X}$ & $\mathrm{X}$ \\
\hline & Digitaria insularis (L.) Fedde & Sourgrass & $\mathrm{X}$ & & $\mathrm{X}$ & $\mathrm{X}$ & $\mathrm{X}$ & $\mathrm{X}$ & \\
\hline & Eleusine indica (L.) Gaertn. & $\begin{array}{l}\text { Indian goosegrass, } \\
\text { wiregrass, or } \\
\text { crowfootgrass } \\
\end{array}$ & $\mathrm{X}$ & $\mathrm{X}$ & $\mathrm{X}$ & $\mathrm{X}$ & $\mathrm{X}$ & $\mathrm{X}$ & $\mathrm{X}$ \\
\hline & Panicum maximum Jacq. & Guinea grass & $\mathrm{X}$ & $\mathrm{X}$ & $\mathrm{X}$ & & $\mathrm{X}$ & $\mathrm{X}$ & \\
\hline & Pennisetum americanum (L.) Leeke & Millet & & & $\mathrm{X}$ & & $\mathrm{X}$ & & \\
\hline & Pennisetum setosum (Sw). Rich. & Fountain grass & $\mathrm{X}$ & $\mathrm{X}$ & $\mathrm{X}$ & & $\mathrm{X}$ & $\mathrm{X}$ & \\
\hline & Rhynchelytrum repens (Willd.) C. E. Hubb & Red Natal grass & & $\mathrm{X}$ & $\mathrm{X}$ & & & & \\
\hline & Setaria parviflora (Poir.) Kerguélen & $\begin{array}{l}\text { Marsh bristlegrass, } \\
\text { bristly foxtail, } \\
\text { knotroot bristlegrass, } \\
\text { or yellow bristlegrass }\end{array}$ & $\mathrm{X}$ & $\mathrm{X}$ & & $\mathrm{X}$ & $\mathrm{X}$ & $\mathrm{X}$ & $\mathrm{X}$ \\
\hline & Sorghum halepense (L.) Pers. & Sorghum & & & & & $\mathrm{X}$ & & \\
\hline & Zea mays L. & Maize & $\mathrm{X}$ & & & & $\mathrm{X}$ & & \\
\hline \multirow{4}{*}{ 4. Smilacaceae } & Smilax brasiliensis Spreng. & $\begin{array}{l}\text { Catbriers, greenbriers, } \\
\text { prickly-ivys, or } \\
\text { smilaxes. }\end{array}$ & $\mathrm{X}$ & & & & & & \\
\hline & Smilax campestris Griseb. & \begin{tabular}{|l|} 
Common names \\
include catbriers, \\
greenbriers, prickly- \\
ivys, and smilaxes \\
\end{tabular} & $\mathrm{X}$ & $\mathrm{X}$ & $\mathrm{X}$ & & & & \\
\hline & Smilax ovolifolia Roxb. & $\begin{array}{l}\text { Common names } \\
\text { include catbriers, } \\
\text { greenbriers, prickly- } \\
\text { ivys, and smilaxes }\end{array}$ & & $\mathrm{X}$ & & & & & \\
\hline & Smilax polyantha Griseb. & \begin{tabular}{|l} 
Common names \\
include catbriers, \\
greenbriers, prickly- \\
ivys, and smilaxes \\
\end{tabular} & & & & & $X$ & & \\
\hline
\end{tabular}

RR soybeans (glyphosate-tolerant soybeans); CV soybeans (conventional soybeans). 
Rubiaceae. Families Amaranthaceae, Convolvulaceae, Cyperaceae, Lamiaceae, Caesalpiniaceae, Myrtaceae and Polygonaceae presented two species each. The other families (13 of the total) contributed with a single species. In sunflower cultivation in Goiás, Adegas et al. (2010) have recorded 41 species distributed in 13 botanical families. Of this total, 24 species were equal to the ones in the present study. For the same crop grown in Brazilian state Rio Grande do Sul, these authors have cataloged 37 species and 15 families, of which 14 species were similar to those of this survey.

Families Asteraceae, Poaceae and Euphorbiaceae were the ones that contributed the largest number of species in sunflower crops in Brazilian municipalities Chapadão do Céu, Jataí and Montividiu, in Goiás (Brighenti et al., 2003, Adegas et al., 2010). These families were also representative in the soybeans rotated with maize and rice in Brazilian state Roraima (Cruz et al., 2009). These latter authors state that Asteraceae and Poaceae are the two main families of existing weeds in the cerrado, being present in different grain production systems, sugarcane, floodplain fields exploitation and pastures.

Regarding the distribution of species per genera, those which have contributed in greater diversity were: Sida, Smilax, Cissampelos, Digitaria, Bidens, Conyza, Ipomoea, Cyperus, Crotalaria, Pennisetum, Rumex and Spermacoce. The other genera, 48 of the total, had only a single species. As for the aforementioned genera, it should be highlighted that there was no mention about the Smilax genus incidence in areas of agricultural crops in the cerrado.

As for the distribution of the number of species per treatment, the combination $\mathrm{RR}$ soybean + millet presented the highest number (44 species), followed by RR soybean + maize (43), RR soybean + sorghum (40), conventional soybean + maize (38), RR soybean + fallow (35), conventional soybean + millet (30) and conventional soybean + sorghum (18) (Figure 1).

It was observed that for glyphosate-tolerant soybeans, regardless of the succession culture, the highest diversity of species was recorded in relation to conventional soybean fields. These data contradict some studies that show that successive applications of glyphosate reduce the diversity of the weed community. However, it is known that, in conventional soybeans, herbicides used are repeated year after year and primarily consist of an associated application of ALS inhibitors with Protox inhibitors, followed by an application of ACCase inhibitor. That is, the characteristics of the species selection factor are only changed.

As for the distribution of species by seasons, the RR soybeans + millet treatment had the highest number of species (39) in the first evaluation (pre-planting desiccation), followed by RR soybean + maize (38), conventional soybean + maize (36), RR soybean + sorghum (34), RR soybean + fallow (31), conventional soybean + millet (27), and conventional soybean + sorghum (16) (Figure 2). In the second evaluation (period prior to the postemergence herbicide application), the RR soybean + maize treatment showed the greatest number of species (16 of the total). Regarding the third evaluation (late harvest), for treatments RR soybean + millet, RR soybean + sorghum and conventional soybean + millet, 14 species were recorded, followed by conventional soybean + maize (13), RR soybean + maize (12), RR soybean + fallow, and conventional soybean + sorghum (11).

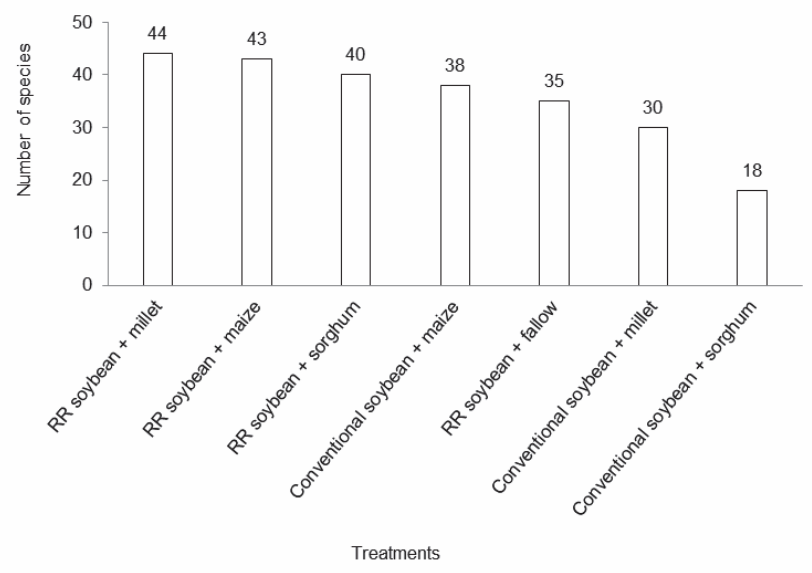

Figure 1 - Number of species (richness) of weeds found in agricultural areas in southwestern Goiás under different production systems. 


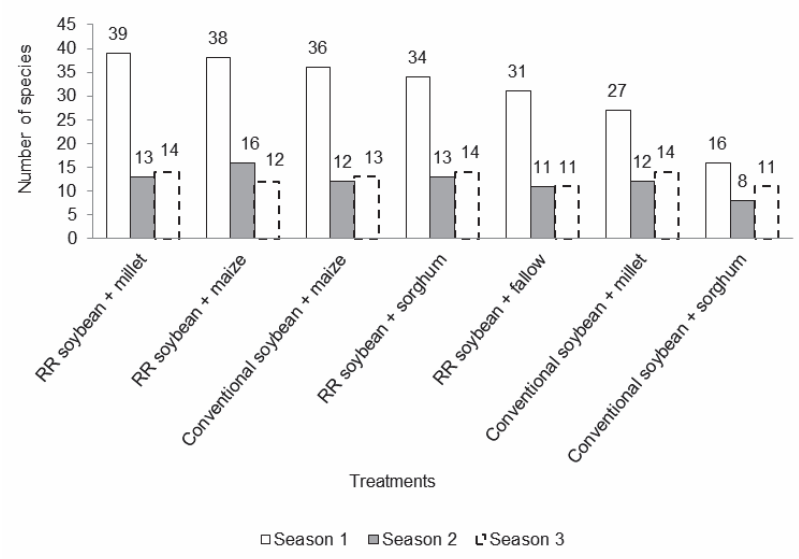

Figure 2 - Number of species (richness) of weeds in agricultural areas in southwestern Goiás under different production systems and different survey seasons.

It is noteworthy that in sorghum, regardless of the type of soybeans and evaluation time, there was less diversity of species. In these areas, the predominance of the C. echinatus species was noted in the period prior to desiccation for soybean seeding. Soil cover by the species in question, along with sorghum stover on the surface, may cause allelopathy (Vidal \& Trezzi, 2004) and has influenced the smaller number of species in the study area. Additionally, sorghum presents limitation of grass herbicides for use in postemergence (Archangelo et al., 2002; Dan et al., 2010), which favors the simultaneous development of the C. echinatus species with the culture, and its spread in the growing area.

Greater diversity of weeds was noted in the first evaluation, which is justified by the longer period without herbicide application. In southwestern Goiás agricultural areas, the absence of cultivation from June/July to October/November because of low rainfall is observed. Furthermore, the stover on the surface from the late harvest cultures in many cases is not sufficient to suppress the emergence of weeds at this time. The species with the highest importance values in the first evaluation were A. tenella, B. subalternans, C. echinatus, C. hirta, C. benghalensis, C. canadensis, E. heterophyla, P. pauciflora, S. glaziovii, and S. rhombifolia.

Although the number of species has decreased in the second evaluation period (main crop), some remained as predominant in the areas analyzed, which stood out in importance: A. tenella, B. subalternans, C. echinatus, C. hirta, C. benghalensis, C. bonariensis, C. difformis, E. indica, E. heterophyla and I. grandifolia.

Other species persisted in the third evaluation (late harvest), behaving as most important: C. echinatus, G. $\max$ (voluntary), $C$. benghalensis, E. indica, D. horizontalis, A. tenella, E. heterophyla, B. subalternans, I. cordifolia, and S. obtusifolia, regardless of the late harvest.

\section{Floristic similarity}

In floristic comparison, it was found that treatments conventional soybean + maize and conventional soybeans and millet had a higher similarity index, with $70 \%$ of the species common to these production systems (Table 6). Twenty-five species were similar in these treatments, as follows: A. tenella, B. subalternans, C. canadensis, G. coarctatum, P. pauciflora, T. procumbens, C. benghalensis, I. cordifolia, I. grandifolia, C. difformis, C. hirta, E. heterophylla, L. nepetifolia, S. obtusifolia, G. max, S. glaziovii, S. rhombifolia, Cissampelos sp2., C. echinatus, D. insularis, E. indica, P. maximum, P. setosum, S. parviflora and $R$. brasiliensis. Treatments RR soybean + millet and conventional soybeans + sorghum were those that showed less similarity (51\% of common species). The similarity among treatments was greater than $50 \%$, which for that index is considered a high percentage (Felfili \& Venturoli, 2000).

It is worth noting that in the floristic comparison, $C$. benghalensis and C. echinatus had wide distribution, as they were present in all treatments in the three evaluation periods. Species A. tenella, B. subalternans, T. procumbens, I. grandifolia, C. difformis, C. hirta, E. heterophylla, S. obtusifolia, G. max, $S$. glaziovii, and E. indica have also had widespread occurrence, being observed in all treatments in at least one of the evaluation times.

It is noteworthy that, from these species common to the seven treatments, $B$. subalternans and C. hirta were present in six treatments in all evaluation periods. 
Table 6 - Sørensen-Dice similarity index (SDI) and diversity - number of species (NSP), Shannon-Wiener index (H'), Simpson diversity index (D') and equitability (E') - for RR soybeans (glyphosate-tolerant soybeans) and CV soybeans (conventional soybeans) in southwestern Goiás

\begin{tabular}{|c|c|c|c|c|c|c|c|c|c|c|c|}
\hline \multicolumn{4}{|c|}{ Diversity } & \multirow[b]{2}{*}{ Treatment } & \multicolumn{7}{|c|}{ Similarity (IS) } \\
\hline NSP & $\mathrm{E}^{\prime}$ & $\mathrm{H}^{\prime}$ & $\mathrm{D}$ & & $\begin{array}{l}\text { RR soybean } \\
+\mathrm{RR} \text { maize }\end{array}$ & $\begin{array}{l}\text { RR soybean } \\
+\mathrm{RR} \text { maize }\end{array}$ & $\begin{array}{l}\text { RR soybean } \\
+\mathrm{RR} \text { maize }\end{array}$ & $\begin{array}{l}\text { RR soybean } \\
+ \text { RR maize }\end{array}$ & $\begin{array}{l}\text { RR soybean } \\
+\mathrm{RR} \text { maize }\end{array}$ & $\begin{array}{l}\text { RR soybean } \\
+ \text { RR maize }\end{array}$ & $\begin{array}{l}\text { RR soybean } \\
+ \text { RR maize }\end{array}$ \\
\hline 43 & 0.85 & 1.86 & 0.79 & $\begin{array}{l}\text { RR soybean }+ \\
\text { maize }\end{array}$ & - & 0.6206 & 0.6265 & 0.6153 & 0.6419 & 0.6849 & 0.5573 \\
\hline 44 & 0.83 & 1.71 & 0.76 & $\begin{array}{l}\text { RR soybean }+ \\
\text { millet }\end{array}$ & - & 0 & 0.6428 & 0.5569 & 0.5609 & 0.6486 & 0.5161 \\
\hline 40 & 0.76 & 1.5 & 0.67 & $\begin{array}{l}\text { RR soybean + } \\
\text { sorghum }\end{array}$ & - & - & 0 & 0.5866 & 0.6666 & 0.6571 & 0.5517 \\
\hline 35 & 0.81 & 1.64 & 0.73 & $\begin{array}{l}\text { RR soybean }+ \\
\text { fallow }\end{array}$ & - & - & - & 0 & 0.6575 & 0.6769 & 0.6792 \\
\hline 38 & 0.78 & 1.49 & 0.68 & $\begin{array}{l}\text { CV soybean }+ \\
\text { maize }\end{array}$ & - & - & - & - & 0 & 0.7058 & 0.5357 \\
\hline 30 & 0.79 & 1.59 & 0.71 & $\begin{array}{l}\text { CV soybean + } \\
\text { millet }\end{array}$ & - & - & - & - & - & 0 & 0.625 \\
\hline 18 & 0.71 & 1.11 & 0.57 & $\begin{array}{l}\text { CV soybean + } \\
\text { sorghum }\end{array}$ & - & - & - & - & - & - & 0 \\
\hline
\end{tabular}

Species A. tenella was found in five treatments (RR soybean + maize, $R R$ soybean + millet, $R R$ soybean + sorghum, RR soybean + fallow, and conventional soybean + millet). On the other hand, Euphorbia heterophylla was observed in four treatments (RR soybean + maize, RR soybean + sorghum, conventional soybean + maize and conventional soybean + sorghum).

Other five species (C. bonariensis, P. pauciflora, S. rhombifolia, D. horizontalis, and $S$. parviflora) were recorded in six of the seven treatments, and A. viridis, C. canadensis, G. coarctatum, I. cordifolia, $M$. coromandelianum, $D$. insularis, $P$. maximum, and $P$. setosum in five treatments, regardless of the evaluation period. Twenty-eight species were endemic to only one treatment, with changes in evaluation periods.

Of the species recorded in this study, it was observed that Benghal dayflower (C. benghalensis), asthma plant, asthma weed, asthmaplant, cat's hair, flowery headed spurge, garden spurge, hairy spurge, Jean Roberts, old blood, pill bearing spurge, pillbearing spurge, pillpod sandmat, pillpod spurge, Queensland asthma weed, red euphorbia, red milkweed, snake weed, snakeweed, sneeze weed, or spurge (Chamaesyce hirta), joyweeds
(Alternanthera tenella), Indian goosegrass, wiregrass, or crowfootgrass (E. indica), (Mexican) fireplant, painted euphorbia, Japanese poinsettia, desert poinsettia, wild poinsettia, fire on the mountain, paintedleaf, painted spurge, milkweed, and kaliko plant (E. heterophylla), morning glory, sweet potato, bindweed, moonflower, etc. (Ipomoea spp.), coat buttons or tridax daisy (T. procumbens), horseweed (Conyza spp.), sourgrass (D. insularis), tropical Mexican clover, Brazilian calla-lily, white-eye, for Brazil pusley (R. brasiliensis), buttonweed (S. latifolia), shrubby false buttonweed (S. verticilata), straggler daisy (S. grisebachii), and beggarticks, black jack, burr marigolds, cobbler's pegs, Spanish needles, stickseeds, tickseeds, or tickseed sunflowers (Bidens spp.) are often occurring in soybean cropping systems. It is noteworthy that in Brazil and other countries such as Argentina and the United States, these species have been reported as tolerant or glyphosate resistant (Papa et al., 2002; Culpepper, 2006; Heap, 2015).

Regarding diversity and equitability (Table 6), these indexes are used to analyze each area or treatment due to the variety of species and for comparison of similarity among treatments. This information provides 
important elements for the understanding of the occurrence of species interactions according to the selection of the management system and the practices in the areas (Concenço et al., 2013).

In this study, soybeans $\mathrm{RR}+$ maize treatment had higher equitability (0.85), which depicts a lower number of dominant species or with high relative abundance, showing greater species variability. Also in this treatment higher values of the ShannonWiener and Simpson diversity indexes were found: 1.86 and 0.79 , respectively.

The cultivation system involving conventional soybean + sorghum had the lowest average values of equitability and differences, showing that a small number of species predominates in these areas. It was expected that fallow sites in the late harvest period presented the highest diversity, which was not observed. This treatment was positioned as intermediate regarding equitability $(0,81)$, Shannon-Wiener $(1,64)$ diversity and Simpson $(0,73)$ for number of individuals. In fallow areas, predominance of species such as southern sandspur or southern sandbur (Cenchrus echinatus) and joyweeds (Alternanthera tenella), covering the soil surface has also been observed.

Assessing five treatments in the Brazilian city of Dourados, MS, Concenço et al. (2013) have found Shannon-Wiener and Simpson indexes in the areas of maize succeeding soybeans of 3.11 and 0.83 , respectively. The diversity data for the maize + signalgrass treatment recorded by these authors and the RR soybean + maize treatment of this study showed similar values for Simpson index (0.79).

Areas with RR soybean crops had greater species diversity in relation to areas with conventional soybeans. Sites with genetically modified soybean in succession with maize had fewer dominant species, i.e., greater diversity. As for sites with conventional soybeans + sorghum in succession, there was low variability, and a few dominant species occurring.

Species C. echinatus and C. benghalensis were the ones of higher incidence. Other
11 species (A. tenella, B. subalternans, T. procumbens, I. grandifolia, C. difformis, C. hirta, E. heterophylla, S. obtusifolia, G. max, S. glaziovii, and E. indica) have also shown widespread occurrence, since they were recorded in $R R$ soybean fields and in conventional soybean sites, irrespective of the type of second crop culture.

Field surveys allowed to verify the qualitative and quantitative differences in the weed communities analyzed. In the glyphosate-tolerant soybeans cultivation sites, a higher number of species was recorded. In the areas of late harvest sorghum, dominance of a small number of species was evident. Unwieldy species were recorded in all farming systems analyzed. These species must be observed prior to chemical, mechanical or cultural management decisions in the study area.

\section{LITERATURE CITED}

ADEGAS, F. S. et al. Levantamento fitossociológico de plantas daninhas na cultura do girassol. Planta Daninha, v. 28 , n. 4 , p. $705-716,2010$.

ARCHANGELO, E. R. et al. Tolerância do sorgo forrageiro ao herbicida Primestra SC. R. Bras. Milho Sorgo, v. 1, n. 2, p. 59-66, 2010.

BALBINOT JR., A. A.; VEIGA, M. Densidade de plantas daninhas afetada por sistemas de manejo do solo e de adubação. R. Ci. Agrovet., v. 13, n. 1, p. 47-55, 2014.

BARROS, M. P. C.; MENDONÇA, C. G.; TROPALDI, L. Controle de plantas daninhas com herbicida glyphosate utilizando diferentes pontas de pulverização. Sci. Agr., v. 15, n. 1, p. 15-21, 2014.

BRAUN-BLANQUET, J. Fitossociologia: bases para el estudio de lãs comunidades vegetales. Madri: H. Blume, 1979. $820 \mathrm{p}$.

BRIGHENTI, A. M. et al. Cadastramento fitossociológico de plantas daninhas na cultura de girassol. Pesq. Agropec. Bras., v. 38, n. 5, p. 651-657, 2003.

CONCENÇO, G. et al. Phytosociological surveys: tools for weed science. Planta Daninha, v. 31, n. 2, p. 469-482, 2013.

CORRÊA M. L. P. et al. Dinâmica populacional de plantas daninhas na cultura do milho em função de adubação e manejo. R. Ci. Agron., v. 42, n. 2, p. 354-363, 2011. 
CRUZ, D. L. S. et al. Levantamento de plantas daninhas em área rotacionada com as culturas da soja, milho e arroz irrigado no cerrado de Roraima. R. Bras. Agroamb, v. 3, n. 1, p. 58-63, 2009.

CULPEPPER, A. S. Glyphosate induced weed shifts. Weed Technol., v. 20, n. 2, p. 277-281, 2006.

DAN, H. A. et al. Tolerância do sorgo granífero ao herbicida tembotrione. Planta Daninha, v. 28, n. 3, p. 615-620, 2010.

FELFILI, J. M.; VENTUROLI, F. Tópicos em análise de vegetação. Comunicações Técn. Flor., v. 2, n. 2, p. 1-25, 2000 .

FIALHO, C. M. T. et al. Fitossociologia da comunidade de plantas daninhas na cultura da soja transgênica sob dois sistemas de preparo do solo. Sci. Agr., v. 12, n. 1, p. 9-17, 2011.

FOLTRAN, R. et al. Levantamento fitossociológico das comunidades de plantas infestantes em diferentes sistemas de rotação de culturas. In: CONGRESSO BRASILEIRO DA CIÊNCIA DAS PLANTAS DANINHAS, 2010, Ribeirão Preto. Anais... Ribeirão Preto: SBCPD, 2010.

HEAP, I. The international survey of herbicide resistant weeds. Disponível em: $<$ http://www.weedscience.com/ summary/MOA. aspx>. Acesso em: 25 ago. 2015.

MCCUNE, B. J.; MEFFORD, M. J. Multivariate analysis of ecological data. PC-ORD Version 6.0, 2011.

MUELLER-DOMBOIS, D.; ELLEMBERG, H. A. Aims and methods of vegetation ecology. New York: John Wiley, 1974. $574 \mathrm{p}$.
PAPA, J. C. M.; FELÍZIA, J. C.; ESTÉBAN, A. J. Cambios em la flora de malezas como consecuencia del cambio tecnologico em Argentina: malezas nove dosas que piedenafectar al cultivo de soja. In: CONGRESSO BRASILEIRO DE SOJA/MERCOSOJA, Londrina, 2002.

Anais... Londrina: Embrapa Soja; 2002. p. 346-354. (Documentos, 180),

PEREIRA, E. S. et al. Avaliações qualitativas e quantitativas de plantas daninhas na cultura da soja submetida aos sistemas de plantio direto e convencional. Planta Daninha, v. 18, n. 2, p. 207-216, 2000.

PITELLI, R. A. Estudos fitossociológicos em comunidades infestantes de agroecossistemas. J. Consherb, v. 1, n. 2, p. 1-7, 2000.

RADOSEVICH, S. R.; HOLT, J.; GHERSA, C. Weed ecology: implications for management. 2. ed. New York: John Wiley \& Sons, 1997. 589 p.

SANTOS, H. G. et al. O novo mapa de solos do Brasil. Rio de Janeiro: Embrapa Solos, 2011. 67 p.

(Documentos, 130)

SORENSEN, T. A. Method of establishing groups of equal amplitude in plant society based on similarity of species content. In: ODUM, E. P. Ecologia. 3. ed. México: Interamericana, 1972. p. 341-405.

VIDAL, R. A.; TREZZI, M. M. Potencial da utilização de coberturas vegetais de sorgo e milheto na supressão de plantas daninhas em condição de campo: I-plantas em desenvolvimento vegetativo. Planta Daninha, v. 22, n. 2, p. 217-233, 2004. 